\title{
Shifting Vital Rate Correlations Alter Predicted Population Responses to Increasingly Variable Environments
}

\author{
David T. Iles, ${ }^{1, \star}$ Robert F. Rockwell, ${ }^{2}$ and David N. Koons ${ }^{3}$ \\ 1. Biology Department, Woods Hole Oceanographic Institution, Woods Hole, Massachusetts 02543; 2. Department of Ornithology, \\ Division of Vertebrate Zoology, American Museum of Natural History, New York, New York 10024; 3. Department of Fish, Wildlife, \\ and Conservation Biology, Colorado State University, Fort Collins, Colorado 80523
}

Submitted October 27, 2017; Accepted August 21, 2018; Electronically published January 7, 2019

Online enhancements: appendixes S1-S4.

\begin{abstract}
Time series of vital rates are often used to construct "environment-blind" stochastic population projections and calculate the elasticity of population growth to increased temporal variance in vital rates. Here, we show that the utility of this widely used demographic tool is greatly limited by shifts in vital rate correlations that occur as environmental drivers become increasingly variable. The direction and magnitude of these shifts are unpredictable without environmentally explicit models. Shifting vital rate correlations had the largest fitness effects on life histories with short to medium generation times, potentially hampering comparative analyses based on elasticities to vital rate variance for a wide range of species. Shifts in vital rate correlations are likely ubiquitous in increasingly variable environments, and further research should empirically evaluate the life histories for which detailed mechanistic relationships between vital rates and environmental drivers are required for making reliable predictions versus those for which summarized demographic data are sufficient.
\end{abstract}

Keywords: covariation, elasticity, demography, fitness, life history, stochasticity.

\section{Introduction}

Climate change has altered the frequency of extreme weather events, and further changes to environmental variability are expected (IPCC 2012). Over the short term, a careful consideration of the effect of environmental variability on demography and population growth is necessary for guiding species conservation actions (Doak et al. 2005; Boyce et al. 2006; Morris et al. 2008). At longer timescales, a clear understanding of the effects of environmental variability on population growth can illuminate the drivers of life-history evolution (Tuljapurkar 1990; Tuljapurkar et al. 2009).

\footnotetext{
* Corresponding author; email: david.thomas.iles@gmail.com. ORCIDs: Iles, http://orcid.org/0000-0002-7251-4938; Rockwell, http:// orcid.org/0000-0001-9394-8623.

Am. Nat. 2019. Vol. 193, pp. E57-E64. (c) 2019 by The University of Chicago. 0003-0147/2019/19303-58037\$15.00. All rights reserved.

DOI: $10.1086 / 701043$
}

Elasticity analysis is a widely used tool for predicting the effects of changes to demographic variation on population performance (Doak et al. 2005; Morris et al. 2008; Dalgleish et al. 2010; Jongejans et al. 2010; Miller et al. 2011). Elasticity to vital rate variance, $E^{\sigma}$, measures the change in stochastic population growth rate caused by a proportional change in the variance of a focal vital rate (Tuljapurkar et al. 2003; Haridas and Tuljapurkar 2005). Accordingly, $E^{\sigma}$ is commonly used to examine the consequences of vital rate variation both within and across populations, providing insight into evolutionary processes and comparative sensitivity to future increases in environmental variance (Morris et al. 2008). Although $E^{\sigma}$ is typically much smaller than elasticity to changes in the mean of demographic parameters, some populations do exhibit high sensitivity to demographic variance (e.g., Haridas and Tuljapurkar 2005; Dalgleish et al. 2010; Haridas et al. 2015). For example, in a comparative analysis of 10 forb species, the magnitude of $E^{\sigma}$ was $48 \%$ as large as $E^{\mu}$ on average for fertility (range: $16 \%-100 \%$ ) and was $20 \%$ as large as $E^{\mu}$ for survival (range: 0\%-100\%; Dalgleish et al. 2010). Furthermore, even if $E^{\sigma}$ is small in magnitude, it can nevertheless be ecologically relevant if demographic variance is more likely to change than mean demographic rates (Tuljapurkar 2010). This could occur, for example, in cases where environmental variance is changing more rapidly than mean environmental conditions (e.g., Mulder et al. 2016).

A major advantage of this tool is that it can make use of relatively coarse and previously published demographic information (i.e., a time series of vital rate estimates). Additionally, stochastic vital rate elasticities can be calculated without explicitly characterizing the underlying environmental drivers of vital rates. Indeed, Crone et al. (2011) found that explicit environmental drivers were included in less than $50 \%$ of the studies that reported stochastic population growth rates. This approach is therefore particularly appealing given the increasing accessibility of large vital rate databases (e.g., Salguero-Gómez et al. 2014, 2016), for which 
this method could be readily applied to identify the populations or life histories that are most sensitive to environmental variation (e.g., Morris et al. 2008; Jongejans et al. 2010; Van Allen et al. 2012; Coutts et al. 2016; Csergő et al. 2017).

Importantly, $E^{\sigma}$ describes the effect of increased vital rate variation on $\lambda_{\mathrm{s}}$ assuming that all other properties of vital rates remain unchanged. However, vital rate correlations will often simultaneously shift as vital rates become more variable (see mentions of this in Boyce et al. [2006], Gotelli and Ellison [2006], and Jenouvrier [2013]). This follows from the fact that vital rate variability is a function of multiple environmental drivers and life-history trade-offs, each contributing differently to correlation and variance in vital rates. Any particular environmental driver is unlikely to explain all of the variance in a vital rate and will thus strengthen, erode, or even reverse existing correlations as it becomes more variable. For example, if two vital rates are linearly affected by a shared environmental driver (and their residual variance is uncorrelated), increased variability in that driver will strengthen the correlation between them (see the illustration in app. S1; apps. S1-S4 are available online). Conversely, increased variability in an unshared variance component in one or both drivers will weaken their overall correlation (app. S1). These shifting vital rate correlations, which are not accounted for by $E^{\sigma}$, could potentially reduce the utility of this common demographic tool and alter predicted responses to increasingly variable environments.

Here, we use a simulation approach to directly evaluate the importance of shifting vital rate correlations in increasingly variable environments. Our analysis quantifies the potential fitness effects of these shifts as well as their resulting implications for using $E^{\sigma}$ as a basis for prediction. We begin by introducing a matrix projection model that links vital rates to time-varying environmental drivers, and we discuss the calculation of $E^{\sigma}$. We then use this population model to examine (1) the capacity of vital rate correlations to shift as an environmental driver becomes more variable, (2) how these shifts impact fitness (measured as $\lambda_{\mathrm{s}}$ ), and (3) the ability of $E^{\sigma}$ to anticipate changes in $\lambda_{\mathrm{s}}$. We additionally examine how life-history tempo influences population responses to shifting vital rate correlations and how these responses differ when one or multiple vital rates are jointly affected by an environmental driver.

\section{A Population Model for Studying the Effects of Increasingly Variable Environments}

In a variable environment, a structured population can be modeled as follows:

$$
\mathbf{n}(t)=\mathbf{A}(t) \mathbf{n}(t-1),
$$

where $\mathbf{n}(t)$ is the $J \times 1$ population vector at time $t$ and $\mathbf{A}(t)$ is the $J \times J$ population projection matrix that describes tran- sitions among $J$ stage, age, or size classes in the population (Caswell 2001). The elements of $\mathbf{A}(t)$ are often functions of lower-level vital rates that also vary through time (Morris and Doak 2004).

In our investigation, we used a simple stage-structured matrix model to characterize population dynamics (Neubert and Caswell 2000):

$$
\mathbf{A}(t)=\left[\begin{array}{cc}
S_{\mathrm{J}}(1-p) & F(t) \\
S_{\mathrm{J}} p & S_{\mathrm{A}}(t)
\end{array}\right] .
$$

The model discriminates between nonreproductive juveniles and reproductive adults and allows vital rates to vary through time. In this model, $S_{\mathrm{A}}(t)$ represents time-specific adult survival and $F(t)$ represents time-specific fertility (i.e., the contribution of adults to the juvenile stage class). The terms $S_{\mathrm{J}}$ and $p$ denote juvenile survival and the maturation probability of juveniles, respectively. For simplicity, $S_{\mathrm{J}}$ and $p$ are time invariant in our investigation, and we focus on temporal covariation in $S_{\mathrm{A}}(t)$ and $F(t)$.

For structured populations (as in eq. [2]), the long-term stochastic population growth rate, $\lambda_{\mathrm{S}}$, can be calculated numerically or can be approximated as follows (Tuljapurkar 1982a; Haridas and Tuljapurkar 2005):

$$
\log \lambda_{\mathrm{S}} \approx \log \lambda_{\mathrm{D}}+\frac{1}{2} \sum_{i j} E_{i j}^{\sigma} .
$$

The term $\lambda_{\mathrm{D}}$ describes deterministic population growth in the absence of vital rate variation. Each $E_{i j}^{\sigma}$ describes the effect on $\lambda_{\mathrm{S}}$ of a proportional increase in the variance of the matrix element (or vital rate) in row $i$, column $j$, independent of other vital rates; Haridas and Tuljapurkar (2005) provided an exact equation (their eq. [2]) for this quantity. Notably, the $E_{i j}^{\sigma}$ can be calculated using a time series of vital rates without explicit reference to the underlying environmental drivers that generate their variation. The sum of matrix element elasticities to variance, $\sum_{i j} E_{i j}^{\sigma}$, is strictly negative and measures the total amount that demographic variation reduces population growth in a variable environment. Simultaneously, $\sum_{i j} E_{i j}^{\sigma}$ measures the effect on $\lambda_{\mathrm{s}}$ if the variances of all matrix elements increase by the same proportional amount and has been used to compare the relative sensitivity of species to future increases in demographic variability (e.g., Morris et al. 2008; Jongejans et al. 2010).

\section{Scenario 1: Increased Environmental Variability Affects a Single Vital Rate}

We used a simulation approach to examine the ability of $E^{\sigma}$ to predict the effect of future increases in demographic variation on population growth (although note that analytical approaches for understanding the fitness effects of both withinand between-year correlations are also possible; Tuljapurkar 
1982b). We first generated 1,000 life histories (using eq. [2]) by randomly selecting values between 0 and 1 for $\bar{S}_{\mathrm{A}}, \bar{S}_{\text {J }}$, and $\bar{p}$. We solved each matrix for $\bar{F}$ such that $\lambda_{\mathrm{D}}=1$ for each population (i.e., stationary abundance in a constant environment). For each life history and for each vital rate $F(t)$ and $S_{\mathrm{A}}(t)$, we chose a random coefficient of variation between 0 and 0.3 . For simplicity, we did not include variation in $S_{\mathrm{J}}$ and $p$. We then generated a time-varying, standard normal environmental driver, $\operatorname{ENV}(t)$, that was linearly related to $S_{\mathrm{A}}(t)$ but not linked to $F(t)$. We allowed $\operatorname{ENV}(t)$ to explain a random proportion (between 0 and 1) of the variation in $S_{\mathrm{A}}(t)$. Finally, we generated a random correlation ranging from -1 to 1 for the residual variance in $S_{A}(t)$ and $F(t)$, for example, caused by other "unexplained" environmental covariates or life-history trade-offs. We generated 1 million values for $\operatorname{ENV}(t)$ and each vital rate in this "reference environment," assuming that vital rates were normally distributed (but see app. S2 for simulations and expanded results where $S_{\mathrm{A}}(t)$ is beta distributed and $F(t)$ is lognormal). We calculated $\lambda_{\mathrm{S}}$ and the corresponding elasticity of $\lambda_{\mathrm{S}}$ to variance in $S_{\mathrm{A}}(t)$, denoted $E_{S_{A}}^{\sigma}$, using eq. (2) in Haridas and Tuljapurkar (2005).

We then investigated whether $E_{S_{\mathrm{A}}}^{\sigma}$ could reliably predict the response of $\lambda_{\mathrm{S}}$ to increased variance in $S_{\mathrm{A}}(t)$. We did so by increasing the variance of $\operatorname{ENV}(t)$ by a sufficient amount to cause a $5 \%$ increase in the standard deviation of $S_{\mathrm{A}}(t)$. While $\operatorname{ENV}(t)$ affected only $S_{\mathrm{A}}(t)$ and did not alter the mean or variance of $F(t)$, this nevertheless could affect the correlation between $S_{\mathrm{A}}(t)$ and $F(t)$ (see app. S1). We calculated $\lambda_{\mathrm{S}}$ in this second, more variable environment. Finally, to isolate the effect of shifting correlations, we generated a third sequence of vital rates where $S_{\mathrm{A}}(t)$ was $5 \%$ more variable than the reference environment, but we manually fixed the correlation at its initial value in the reference environment (see the expanded description of simulation methods in app. S1). If any $S_{\mathrm{A}}(t)$ was greater than 1 or any vital rate less than 0 in any simulation for a life history, we generated a new life history and reran the simulations (see also app. S2). We then evaluated whether $E_{S_{\mathrm{A}}}^{\sigma}$ predicted the resulting change in $\lambda_{\mathrm{S}}$ caused by a $5 \%$ increase in the standard deviation of $S_{\mathrm{A}}(t)$, either when correlations were free to naturally shift or when they were artificially fixed. Finally, because the fitness effects of vital rate correlations are known to depend on generation time (Tuljapurkar et al. 2009), we examined whether the generation times of life histories could explain differences between the change in $\lambda_{\mathrm{s}}$ when correlations were free to shift and when correlations were artificially fixed.

In this scenario, an increase in the standard deviation of an environmental driver generated a $5 \%$ increase in the standard deviation of adult survival and altered $\operatorname{Cor}\left(F, S_{\mathrm{A}}\right)$ for many life histories (shading in fig. $1 A$ ). This shift in vital rate correlations reduced the ability of $E_{S_{\mathrm{A}}}^{\sigma}$ to reliably predict changes in $\lambda_{\mathrm{S}}$ (fig. $1 A$ ). Conversely, $E_{S_{\mathrm{A}}}^{\sigma}$ was almost perfectly predictive of the proportional change in $\lambda_{\mathrm{S}}$ when we artificially fixed the correlation between vital rates (i.e., $R^{2}=1.000$; fig. $1 B$ ). Notably, several life histories had positive values of $E_{S_{\mathrm{A}}}^{\sigma}$ in the reference environment (fig. $1 B$ ), implying that increased variance in $S_{\mathrm{A}}(t)$ should increase $\lambda_{\mathrm{s}}$. Yet none of these life histories actually experienced an increase in $\lambda_{\mathrm{S}}$ following an increase in the variance of $S_{\mathrm{A}}(t)$, owing to shifts toward more positive vital rate correlations, which reduces population growth in variable environments (Doak et al. 2005). Shifts in vital rate correlations occurred for life histories with any generation time, but these shifts had the largest fitness effects for life histories with short to medium generation times (fig. 1C).

\section{Scenario 2: Increased Environmental Variability Affects Multiple Vital Rates}

We conducted a second series of simulations to examine the effects of joint vital rate responses to an increasingly variable environmental driver (see the expanded description of these simulation methods in app. S1). We first generated a linear relationship between $\operatorname{ENV}(t)$ and both $S_{\mathrm{A}}(t)$ and $F(t)$. In these simulations, $\operatorname{ENV}(t)$ explained the same proportion of variance in both $S_{\mathrm{A}}(t)$ and $F(t)$ within a life history, but this proportion differed between life histories (see app. S3 for results when the proportion of variance explained differs among vital rates). Thus, some life histories experienced strong joint responses to $\operatorname{ENV}(t)$ while others experienced weak joint responses. We again chose a random correlation for the residual variance in $S_{\mathrm{A}}(t)$ and $F(t)$ and generated a sequence of 1 million vital rates in the reference environment.

Because $\operatorname{ENV}(t)$ explains the same proportion of variation in both vital rates (and is linearly related to both), an increase in variance in $\operatorname{ENV}(t)$ causes the same proportional increase in variance of both vital rates simultaneously. As in scenario 1 , for each life history we increased the variance of $\operatorname{ENV}(t)$ by an amount resulting in a 5\% increase in the standard deviation of both vital rates and generated a new series of more variable vital rates. We then generated a third sequence of vital rates where $S_{\mathrm{A}}(t)$ and $F(t)$ were $5 \%$ more variable than the reference environment, but their correlation was fixed at its initial value in the reference environment.

We calculated $E^{\sigma}$ for each vital rate in the reference environment and used $\sum_{i j} E_{i j}^{\sigma}$ as a measure of total elasticity to demographic variance. This metric is appropriate for quantifying the response of a population to a proportional increase in the variance of all vital rates simultaneously (Morris et al. 2008), as occurred in this scenario. We then evaluated whether $\sum_{i j} E_{i j}^{\sigma}$ predicted the resulting changes in $\lambda_{\mathrm{S}}$ caused by a $5 \%$ increase in the variability of $S_{\mathrm{A}}(t)$ and $F(t)$.

In this scenario, the joint effect of $\operatorname{ENV}(t)$ on $F(t)$ and $S_{\mathrm{A}}(t)$ more strongly altered vital rate correlations than if only a single vital rate responded to the environmental driver (the range of $\Delta \operatorname{Cor}\left(F, S_{\mathrm{A}}\right)$ is larger in fig. $2 A$ than in fig. $1 A$ ). This further reduced the ability of vital rate elasticities to reliably 


\section{A}

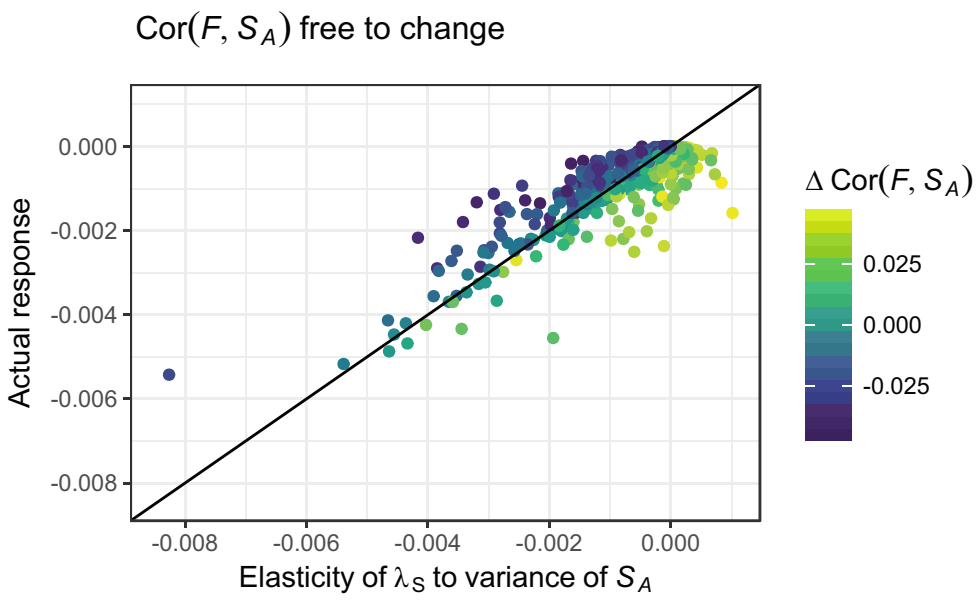

B $\quad \operatorname{Cor}\left(F, S_{A}\right)$ artificially fixed at initial value

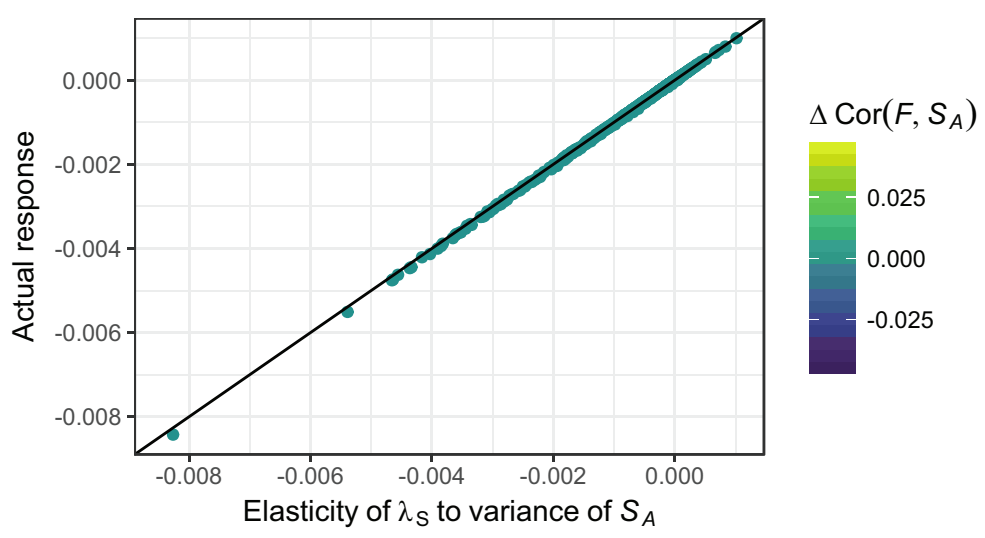

C

Difference Between Responses (Effect of shifting vital rate correlations)

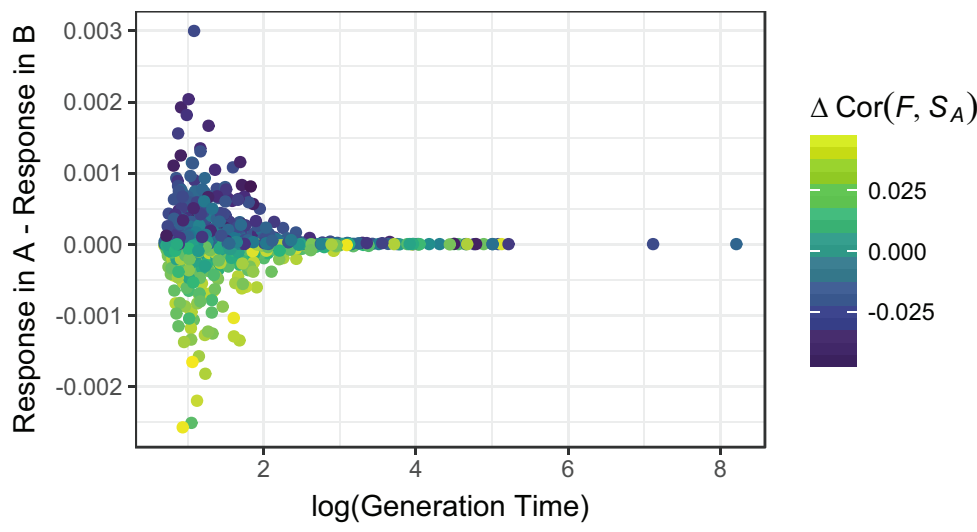

Figure 1: Relationship between the elasticity to variance in adult survival, $E_{S_{\mathrm{A}}}^{\sigma}$ (calculated in a reference environment), and the actual proportional change in $\lambda_{\mathrm{S}}$ that occurs when the standard deviation of adult survival increases by $5 \%$, calculated numerically using $\Delta \lambda_{\mathrm{S}} / \lambda_{\mathrm{S}} \times$ $\operatorname{sd}\left(S_{\mathrm{A}}\right) / \Delta \operatorname{sd}\left(S_{\mathrm{A}}\right)$. A, Relationship when vital rate correlations are free to shift naturally $\left(R^{2}=0.805\right)$. The black line represents a perfect $1: 1$ relationship. $B$, Relationship when vital rate correlations are artificially fixed $\left(R^{2}=1.000\right)$. $C$, Differences between responses in $A$ and $B$, plotted against each life history's generation time. While increasing variability of an environmental driver caused vital correlations to shift for all life histories (colored shading in $A$ and $C$ ), these shifts had the largest fitness effects for life histories with short to medium generation times. 
A

$\operatorname{Cor}\left(F, S_{A}\right)$ free to change
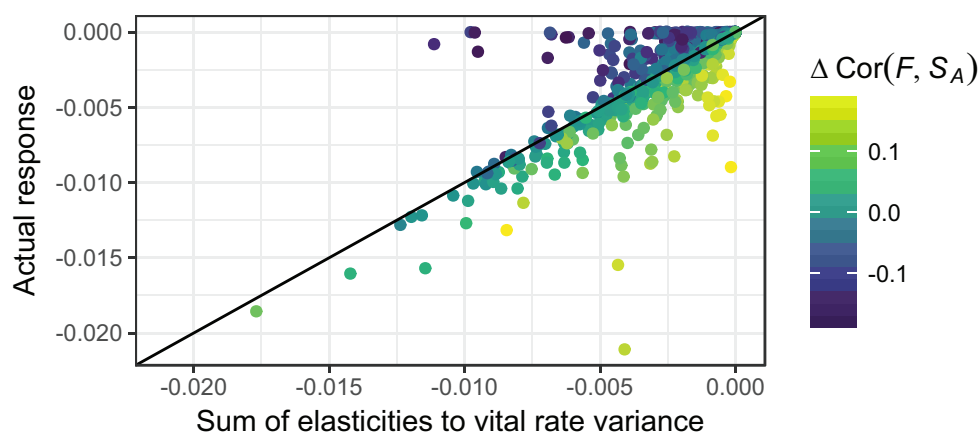

B $\quad \operatorname{Cor}\left(F, S_{A}\right)$ artificially fixed at initial value

$E_{S_{A}}^{\sigma}+E_{F}^{\sigma}$

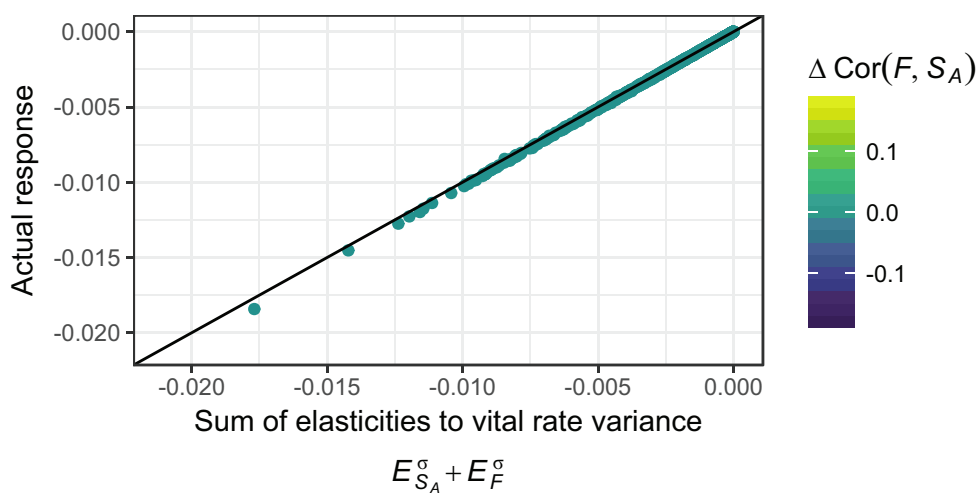

C

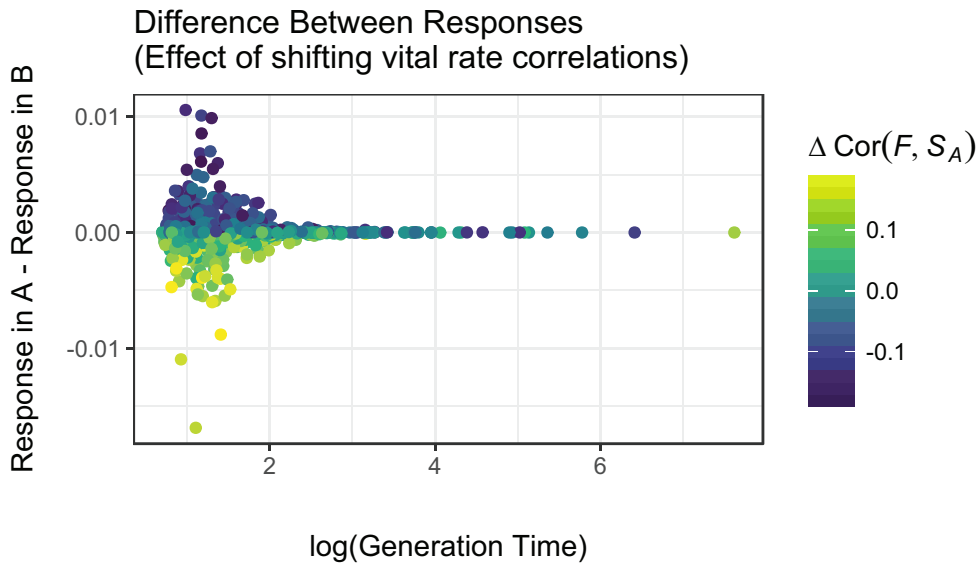

Figure 2: Relationship between the sum of elasticity to variance across two vital rates, $E_{F}^{\sigma}+E_{S_{\mathrm{A}}}^{\sigma}$ (calculated in an initial reference environment) and the actual proportional change in $\lambda_{\mathrm{S}}$ that occurs when the standard deviation of both $S_{\mathrm{A}}(t)$ and $F(t)$ increases by $5 \%$, calculated numerically using $\Delta \lambda_{\mathrm{S}} / \lambda_{\mathrm{S}} \times(1 / 2)\left(\operatorname{sd}\left(S_{\mathrm{A}}\right) / \Delta \mathrm{sd}\left(S_{\mathrm{A}}\right)+\operatorname{sd}(F) / \Delta \operatorname{sd}(F)\right)$. In this scenario the environmental driver explained the same proportion of variation in both vital rates, but this proportion differed among life histories. $A$, Relationship when vital rate correlations are free to shift naturally $\left(R^{2}=0.694\right)$. The black line represents a perfect $1: 1$ relationship. $B$, Relationship when vital rate correlations are artificially fixed $\left(R^{2}=1.000\right)$. $C$, Differences between responses in $A$ and $B$, plotted against each life history's generation time. Note that while shifts in vital rate correlations occurred for all life histories (colored shading in $A$ and $C$ ), these shifts had the largest fitness effects for life histories with short to medium generation times. 
predict changes in $\lambda_{\mathrm{S}}$ as vital rates became more variable (fig. $2 A ; R^{2}=0.694$ ). Vital rate elasticities predicted population responses reliably only when we artificially fixed vital rate correlations to their initial value (fig. $2 B ; R^{2}=1.000$ ). Shifts in vital rate correlations again occurred for life histories across the entire range of generation times, but these shifts had disproportionately large fitness effects for life histories with short to medium generation times (fig. $2 C$ ). We note that these simulations assumed normally distributed vital rate error, which limited the range of variation we could impose without resulting in impossible values for vital rates (e.g., survival rates less than 0 or greater than 1). However, we found similar patterns in a second set of analyses where we used copulas to generate correlated changes in the variance of vital rates, treating survival as a beta random variable and fertility as lognormal, confirming that these results are robust across a much wider range of vital rate variation (app. S2; see examples and further descriptions of this approach in Koons et al. [2008] and de Valpine et al. [2014]).

\section{Conclusions}

We found, somewhat paradoxically, that analyses designed to measure the sensitivity of populations to demographic variance may often be unable to reliably characterize the true effects that occur when vital rate variance responds to an increasingly variable environmental driver. In our study, increased variance in vital rates was driven by increased variance of an underlying environmental driver. The disconnect between $E^{\sigma}$ and the true effect on $\lambda_{\mathrm{S}}$ occurs when the environmental driver explains only a portion of the (co)variance in one or more vital rates, which causes vital rate correlations to shift as the driver (and thus vital rates) become more variable-an important detail that has been mentioned but not closely examined (Boyce et al. 2006; Gotelli and Ellison 2006; Jenouvrier 2013). Vital rate correlations, in turn, affect population growth and sensitivity to further changes in demographic variance (Tuljapurkar 1990; Doak et al. 2005; Tuljapurkar et al. 2009). Given that any particular environmental driver will not explain all of the (co)variation in vital rates (Møller and Jennions 2002), concurrent changes in vital rate variance and correlations are likely ubiquitous. This represents an important and underappreciated limitation of this widely used demographic tool, potentially hampering inferential extensions of $E^{\sigma}$ to real-world applications. Furthermore, vital rates will also often have nonlinear relationships with environmental drivers (Koons et al. 2009; Lawson et al. 2015), such that increased environmental variability affects the mean, variance, and correlations of vital rate distributions simultaneously.

Our results reemphasize the potentially important role of within-year vital rate correlations in variable environments, which are well understood from demographic theory (Tul- japurkar 1982b, 1990; Doak et al. 2005). For example, we found that shifts in vital rate correlations were capable of fully offsetting (or even reversing) the fitness effects of increased demographic variation. Simultaneously, these shifts in vital rate correlations had the largest fitness effects - and thus potentially impact the reliability of $E^{\sigma}$ most strongly-for life histories with short to medium generation times (figs. $1 C$, $2 C)$. Conversely, while shifts in vital correlations did occur for life histories with long generation times, they did not strongly impact fitness, suggesting that elasticity to vital rate variance is quite reliable for these species. These results are consistent with a theoretical analysis by Tuljapurkar et al. (2009) that detected disproportionately strong effects of vital rate correlations on life histories with shorter generation times. The analysis of Tuljapurkar et al. (2009) did not make explicit distributional assumptions about vital rates or the environmental drivers thereof, suggesting that our results are likely robust across a wide array of vital rate distributions (app. S2) and environmental responses.

The fitness effect of a change in the correlation between two vital rates is proportional to the product of the sensitivities and standard deviations of both correlated vital rates: $\bar{S}_{v_{i}} \bar{S}_{v_{j}} \sigma_{v_{i}} \sigma_{v_{j}}$ (see full eq. [6b] in Doak et al. 2005). While there is a maximum upper bound on variance that occurs for "zeroto-one" vital rates such as survival (Morris and Doak 2004), the variance of fertility $\left(\sigma_{F}^{2}\right)$ is not constrained in the same way and tends to be positively related to mean fertility $(\bar{F})$, both in our simulations and in real populations (e.g., see fig. S1.1 in Jongejans et al. 2010). The quantity $\sigma_{S_{\mathrm{A}}} \sigma_{F}$ is therefore largest for life histories with short to medium generation times that have high values of $\bar{F}$. Simultaneously, vital rate sensitivities are not explicit functions of their mean (Morris and Doak 2004), and the quantity $\bar{S}_{S_{\mathrm{A}}} \bar{S}_{F}$ is only weakly correlated with generation time $\left(R^{2}=0.20\right.$; fig. S7). As a result, life histories with short generation times (and large values of $\bar{F}$ ) are disproportionately sensitive to shifts in vital rate correlations (app. S4). This also explains why the magnitude of these effects was much larger when we simulated larger variation in $S_{\mathrm{A}}(t)$ and $F(t)$ by treating each vital rate as beta and lognormal random processes, respectively, rather than Gaussian processes (see app. S2).

Also consistent with theory (Tuljapurkar 1982b; Doak et al. 2005; Tuljapurkar et al. 2009), we found that shifts toward more negative correlations attenuated the reductions in $\lambda_{\mathrm{s}}$ that occurred as environments became more variable. Empirical studies have reported both strong (Coulson et al. 2005; Ezard et al. 2006) and weak (Davison et al. 2013; Compagnoni et al. 2016) contributions of vital rate correlations to time-varying population growth. Our study adds an important layer of insight by showing that vital rate correlations will almost certainly shift as environments become more variable, which can either amplify or dampen the effects of environmental change across a wide range of life his- 
tories (although not for those with extremely long generation times). This suggests that even if vital rate correlations or their effects are currently negligible, they could become increasingly relevant as environments become more variable.

Environmentally explicit population models will be needed to predict the resulting effects on population growth when an environmental factor affects vital rate variances, correlations, and other statistical moments simultaneously. This would ideally entail three sequential steps (Morris et al. 2008; Jenouvrier 2013): (1) characterizing the relationships between vital rates and important environmental drivers along with unexplained (co)variation in vital rates, (2) projecting a series of future environmental conditions according to a particular model of environmental change, and (3) creating a sequence of future vital rates by mapping each vital rate onto the projected environmental conditions while accounting for unexplained vital rate (co)variation. This "environmentally explicit" approach is capable of accounting for environmentally driven changes in multiple statistical moments of vital rate distributions (e.g., if vital rate reaction norms are nonlinear) along with shifts in vital rate correlation structures. Unfortunately, this is not yet feasible for most populations because characterizing the relationships between environmental drivers and all vital rates simultaneously is statistically challenging and data intensive (Schurr et al. 2012; Ehrlén and Morris 2015; Teller et al. 2016; Van de Pol et al. 2016; Lee 2017).

Alternatively, Lee (2017) developed a method to evaluate the elasticity of population growth to changes in the intensity of an environmental driver even when explicit demographic responses to the environmental driver are not known. This approach accounts for the multidimensional demographic shifts (including vital rate correlations) that occur under different environmental regimes and may be particularly useful in cases where the responses of individual vital rates to environmental drivers cannot be reliably estimated. Additionally, demographic responses of well-studied populations could potentially be used to infer the responses of poorly studied populations, although geographic and phylogenetic extrapolations of demography have been met with mixed results (Buckley et al. 2010; Coutts et al. 2016). Currently, comparative analyses of demographic responses to environmental drivers are rare (but see examples in Doak and Morris 2010; Treurnicht et al. 2016). Further research, especially empirical studies, that seek to identify the cases in which summarized environment-blind demographic analyses (including $E^{\sigma}$ ) are sufficient for approximating population responses to increasingly variable environments will therefore be invaluable for guiding ongoing demographic monitoring and species conservation.

\section{Acknowledgments}

D.T.I. received support from Ducks Unlimited Canada, the S. J. and Jesse E. Quinney Foundation, Utah State University, the Frank M. Chapman Memorial Fund, and California Waterfowl. D.N.K. is supported by a James C. Kennedy Endowed Chair of Wetland and Waterfowl Conservation. We thank the editors and anonymous reviewers whose insightful comments greatly improved our study.

\section{Literature Cited}

Boyce, M. S., C. V. Haridas, and C. T. Lee. 2006. Demography in an increasingly variable world. Trends in Ecology and Evolution 21:141148.

Buckley, Y. M., S. Ramula, S. P. Blomberg, J. H. Burns, E. E. Crone, J. Ehrlén, T. M. Knight, J.-B. Pichancourt, H. Quested, and G. M. Wardle. 2010. Causes and consequences of variation in plant population growth rate: a synthesis of matrix population models in a phylogenetic context. Ecology Letters 13:1182-1197.

Caswell, H. 2001. Matrix population models: construction, analysis and interpretation. Sinauer, Sunderland, MA.

Compagnoni, A., A. J. Bibian, B. M. Ochocki, H. S. Rogers, E. L. Schultz, M. E. Sneck, B. D. Elderd, et al. 2016. The effect of demographic correlations on the stochastic population dynamics of perennial plants. Ecological Monographs 84:480-494.

Coulson, T., J. M. Gaillard, and M. Festa-Bianchet. 2005. Decomposing the variation in population growth into contributions from multiple demographic rates. Journal of Animal Ecology 74:789801.

Coutts, S. R., R. Salguero-Gómez, A. M. Csergő, and Y. M. Buckley. 2016. Extrapolating demography with climate, proximity and phylogeny: approach with caution. Ecology Letters 19:1429-1438.

Crone, E. E., E. S. Menges, M. M. Ellis, T. Bell, P. Bierzychudek, J. Ehrlén, T. N. Kaye, et al. 2011. How do plant ecologists use matrix population models? Ecology Letters 14:1-8.

Csergő, A. M., R. Salguero-Gómez, O. Broennimann, S. R. Coutts, A. Guisan, A. L. Angert, E. Welk, et al. 2017. Less favourable climates constrain demographic strategies in plants. Ecology Letters 20:969-980.

Dalgleish, H. J., D. N. Koons, and P. B. Adler. 2010. Can life-history traits predict the response of forb populations to changes in climate variability? Journal of Ecology 98:209-217.

Davison, R., F. Nicolè, H. Jacquemyn, and S. Tuljapurkar. 2013. Contributions of covariance: decomposing the components of stochastic population growth in Cypripedium calceolus. American Naturalist 181:410-420.

de Valpine, P., K. Scranton, J. Knape, K. Ram, and N. J. Mills. 2014. The importance of individual developmental variation in stagestructured population models. Ecology Letters 17:1026-1038.

Doak, D. F., and W. F. Morris. 2010. Demographic compensation and tipping points in climate-induced range shifts. Nature 467:959-962.

Doak, D. F., W. F. Morris, C. Pfister, B. E. Kendall, and E. M. Bruna. 2005. Correctly estimating how environmental stochasticity influences fitness and population growth. American Naturalist 166:E14-E21.

Ehrlén, J., and W. F. Morris. 2015. Predicting changes in the distribution and abundance of species under environmental change. Ecology Letters 18:303-314.

Ezard, T. H. G., P. H. Becker, and T. Coulson. 2006. The contributions of age and sex to variation in common tern population growth rate. Journal of Animal Ecology 75:1379-1386.

Gotelli, N. J., and A. M. Ellison. 2006. Forecasting extinction risk with nonstationary matrix models. Ecological Applications 16:51-61. 
Haridas, C. V., K. H. Keeler, and B. Tenhumberg. 2015. Variation in the local population dynamics of the short-lived Opuntia macrorhiza (Cactaceae). Ecology 96:800-807.

Haridas, C., and S. Tuljapurkar. 2005. Elasticities in variable environments: properties and implications. American Naturalist 166:481-495.

IPCC (Intergovernmental Panel on Climate Change). 2012. Managing the risks of extreme events and disasters to advance climate change adaptation. Special report. Cambridge University Press, Cambridge.

Jenouvrier, S. 2013. Impacts of climate change on avian populations. Global Change Biology 19:2036-2057.

Jongejans, E., H. De Kroon, S. Tuljapurkar, and K. Shea. 2010. Plant populations track rather than buffer climate fluctuations. Ecology Letters 13:736-743.

Koons, D. N., C. J. E. Metcalf, and S. Tuljapurkar. 2008. Evolution of delayed reproduction in uncertain environments: a life-history perspective. American Naturalist 172:797-805.

Koons, D. N., S. Pavard, A. Baudisch, and J. E. Metcalf. 2009. Is lifehistory buffering or lability adaptive in stochastic environments? Oikos 118:972-980.

Lawson, C. R., Y. Vindenes, L. Bailey, and M. Pol. 2015. Environmental variation and population responses to global change. Ecology Letters 18:724-736.

Lee, C. T. 2017. Elasticity of population growth with respect to the intensity of biotic or abiotic driving factors. Ecology 98:1016-1025.

Miller, D. A., W. R. Clark, S. J. Arnold, and A. M. Bronikowski. 2011 Stochastic population dynamics in populations of western terrestrial garter snakes with divergent life histories. Ecology 92:1658-1671.

Møller, A., and M. D. Jennions. 2002. How much variance can be explained by ecologists and evolutionary biologists? Oecologia 132:492500 .

Morris, W. F., and D. F. Doak. 2004. Buffering of life histories against environmental stochasticity: accounting for a spurious correlation between the variabilities of vital rates and their contributions to fitness. American Naturalist 163:579-590

Morris, W. F., C. A. Pfister, S. Tuljapurkar, C. V. Haridas, C. L. Boggs, M. S. Boyce, E. M. Bruna, et al. 2008. Longevity can buffer plant and animal populations against changing climatic variability. Ecology 89:19-25.

Mulder, C. P., D. T. Iles, and R. F. Rockwell. 2016. Increased variance in temperature and lag effects alter phenological responses to rapid warming in a subarctic plant community. Global Change Biology 23:801-814.

Neubert, M. G., and H. Caswell. 2000. Demography and dispersal: calculation and sensitivity analysis of invasion speed for structured populations. Ecology 81:1613-1628.
Salguero-Gómez, R., O. R. Jones, C. R. Archer, C. Bein, H. Buhr, C. Farack, F. Gottschalk, et al. 2016. COMADRE: a global data base of animal demography. Journal of Animal Ecology 85:371-384.

Salguero-Gómez, R., O. R. Jones, C. R. Archer, Y. M. Buckley, J. CheCastaldo, H. Caswell, D. Hodgson, et al. 2014. The COMPADRE Plant Matrix Database: an open online repository for plant demography. Iournal of Ecology 103:202-218.

Schurr, F. M., J. Pagel, J. S. Cabral, J. Groeneveld, O. Bykova, R. B. O'Hara, F. Hartig, et al. 2012. How to understand species' niches and range dynamics: a demographic research agenda for biogeography. Journal of Biogeography 39:2146-2162.

Teller, B. J., P. B. Adler, C. B. Edwards, G. Hooker, and S. P. Ellner. 2016. Linking demography with drivers: climate and competition. Methods in Ecology and Evolution 7:171-183.

Treurnicht, M., J. Pagel, K. J. Esler, A. Schutte-Vlok, H. Nottebrock, T. Kraaij, A. G. Rebelo, and F. M. Schurr. 2016. Environmental drivers of demographic variation across the global geographical range of 26 plant species. Journal of Ecology 104:331-342.

Tuljapurkar, S. 1982a. Population dynamics in variable environments. II. Correlated environments, sensitivity analysis and dynamics. Theoretical Population Biology 21:114-140.

- 1982b. Population dynamics in variable environments. III. Evolutionary dynamics of $r$-selection. Theoretical Population Biology 21:141-165.

1990. Population dynamics in variable environments. New York, Springer.

2010. Environmental variance, population growth and evolution. Journal of Animal Ecology 79:1-3.

Tuljapurkar, S., J.-M. Gaillard, and T. Coulson. 2009. From stochastic environments to life histories and back. Philosophical Transactions of the Roval Societv B 364:1499-1509.

Tuljapurkar, S., C. C. Horvitz, and J. B. Pascarella. 2003. The many growth rates and elasticities of populations in random environments. American Naturalist 162:489-502.

Van Allen, B. G., A. E. Dunham, C. M. Asquith, and V. H. Rudolf. 2012. Life history predicts risk of species decline in a stochastic world. Proceedings of the Roval Society B 279:2691-2697.

Van de Pol, M., L. D. Bailey, N. McLean, L. Rijsdijk, C. R. Lawson, and L. Brouwer. 2016. Identifying the best climatic predictors in ecology and evolution. Methods in Ecology and Evolution 7:1246-1257.

Associate Editor: Charlotte T. Lee Editor: Yannis Michalakis 